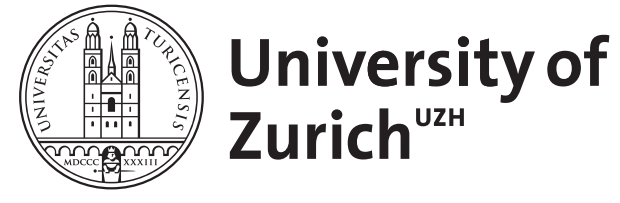

\title{
Werner Jaeger, der Dritte Humanismus und Italien
}

\author{
Näf, Beat
}

DOI: https://doi.org/10.1515/9783110468717-010

Posted at the Zurich Open Repository and Archive, University of Zurich

ZORA URL: https://doi.org/10.5167/uzh-137671

Book Section

Published Version

Originally published at:

Näf, Beat (2017). Werner Jaeger, der Dritte Humanismus und Italien. In: Albrecht, Andrea; Danneberg, Lutz; De Angelis, Simone. Die akademische "Achse Berlin-Rom"? Der wissenschaftlich-kulturelle Austausch zwischen Italien und Deutschland 1920 bis 1945. Berlin, Boston: De Gruyter, 203-228.

DOI: https://doi.org/10.1515/9783110468717-010 


\section{Werner Jaeger, der Dritte Humanismus und Italien}

\section{Einleitung}

Mussolini hat die >Achse Berlin-Rom` am 1. November 1936 in Mailand ausgerufen. In der Folge koordinierten Hitler und Mussolini die Politik ihrer diktatorisch regierten Länder zum Teil tatsächlich in ungewöhnlicher Weise. Man kann in der Schaffung der >Achse Berlin-Rom` etwas historisch Einschneidendes sehen; ein Ereignis, das den Rang eines historischen Datums einnimmt. Doch eigentlich geht das zu weit. Denn offensichtlich haben Gemeinsamkeiten und Verbindungen zwischen Nationalsozialismus und Faschismus für den ganzen Zeitraum der 1920er bis 1940er Jahren eine wichtige Rolle gespielt. Sie sind auch für den wissenschaftlich-kulturellen Austausch zwischen Deutschland und Italien von grundsätzlicher Bedeutung gewesen und haben wohl mehr Einfluss ausgeübt als das Zustandekommen der >Achse Berlin-Rom`.

Was bedeutet dies für die Bewegung des Dritten Humanismus und was ist dieser Dritte Humanismus? Der Dritte Humanismus ist ein internationales und auch nach Italien ausstrahlendes, aber doch zugleich wesentlich deutsches Phänomen, mit Affinitäten zu den Wurzeln des Humanismus in Italien und im antiken Rom. Die Autorität des Humanismus hat auch der Faschismus für sich auszunutzen versucht.

Deshalb stellen sich folgende Fragen. 1) Ist der sogenannte Dritte Humanismus in die Fahrwasser des Faschismus und des Dritten Reiches geraten? 2) Gibt es Gemeinsamkeiten zwischen dem an der Antike einmal mehr entflammten geradezu rauschhaften Bildungsoptimismus und der politisch instrumentalisierten und verzerrten Klassik des Faschismus sowie den inhumanen NSErziehungs- und Züchtungsidealen? 3) Ist es vielleicht gerade umgekehrt so, dass man mit dem Dritten Humanismus dem heraufkommenden Dritten Reich etwas Besseres - eben: einen Dritten Humanismus - entgegen zu stellen versuchte, wobei dieses Bessere vielleicht nur in Emigration und Rückzug verwirklicht werden konnte? 4) Vor allem geht es um die in diesem Beitrag im Hauptteil beantwortete Frage nach der Rezeption des Dritten Humanismus in Italien sowie nach dem Verhältnis zwischen Drittem Humanismus, humanistischen Traditionen und Faschismus. Dabei werden Parallelen und Unterschiede zwischen Deutschland und Italien deutlich. Vor allem wird es um Exponenten der Wis- 
senschaften vom Altertum gehen. Wie haben die Beziehungen zwischen den deutschen Vertretern des Dritten Humanismus und Wissenschaftlern in Italien ausgesehen? Diese Beziehungen erstrecken sich natürlich über die Zeit der 20er bis 40er Jahre hinaus, und da die nach 1945 von ihnen entworfenen Bilder unsere Wahrnehmung der Verhältnisse in der Zeit von Faschismus und Nationalsozialismus beeinflussen, müssen sie mitberücksichtigt werden.

Wir haben es mit einem weiten, großen und wichtigen Thema zu tun. Ich schränke es ein, indem ich mich auf den wichtigsten Repräsentanten des Dritten Humanismus, Werner Jaeger (1888-1961), konzentriere. ${ }^{1}$

Zunächst aber noch einmal zu den größeren Zusammenhängen. Zweifellos sehen wir unser Thema geprägt durch eigene Erfahrungen der Auseinanderset-

\footnotetext{
1 Als Ausgangspunkt mag man vielleicht einen entsprechenden Artikel des Jaeger-Schülers William M. Calder III nehmen: "Werner Jaeger«, in: Geschichte der Altertumswissenschaften. Biographisches Lexikon. (Der Neue Pauly. Supplemente 6), hg. v. Peter Kuhlmann und Helmuth Schneider. Stuttgart, Weimar 2012, S. 617-621 (Calder hat sich wiederholt mit Jaeger auseinandergesetzt). Siehe zusätzlich auch die Ausführungen unter anderem bei Anthony Andurand: ")Jeter un pont entre la science et la vieく. Werner et le troisième humanisme«, in: Kentron 25 (2009), S. 53-76; ders.: »Le Troisième humanisme est-il un classicisme? Werner Jaeger et la question des 'valeurs` de l'Antiquité grecque«, in: Revue germanique internationale 14 (2011), S. 209-224; ders.: Le mythe grec allemand. Histoire d'une affinité élective. Rennes 2014; Johann Chapoutot: Le national-socialisme et l'Antiquité. Paris 2008 (deutsch 2014); Katie Fleming: »Heidegger, Jaeger, Plato: The Politics of Humanism«, in: International Journal of the Classical Tradition 19 (2012), S. 82-106; Andrea Follak: Der »Aufblick zur Idee«. Eine vergleichende Studie zur Platonischen Pädagogik bei Friedrich Schleiermacher, Paul Natorp und Werner Jaeger. Göttingen 2005; Altertumswissenschaft in den 20er Jahren. Neue Fragen und Impulse, hg. v. Hellmut Flashar unter Mitarbeit von Sabine Vogt. Stuttgart 1995 (Aus den Arbeitskreisen »Methoden der Geisteswissenschaften« der Fritz Thyssen Stiftung); Hans Peter Obermayer: Deutsche Altertumswissenschaftler im amerikanischen Exil. Eine Rekonstruktion. Berlin u. a. 2014; Wolfgang Schadewaldt: Gedenkrede auf Werner Jaeger 1888-1961. Mit einem Verzeichnis der Schriften Werner Jaegers. Berlin 1963; Ernst A. Schmidt: »Werner Jaegers `Dritter Humanismus . Analyse des Programmes, der Stimmen der Kritiker und ihrer Positionen «, in: Internationales Jahrbuch für Hermeneutik 2 (2003), S. 193-223; Hellmut Sichtermann: Kulturgeschichte der Klassischen Archäologie. München 1996; Barbara Stiewe: Der „Dritte Humanismus«. Aspekte deutscher Griechenrezeption vom George-Kreis bis zum Nationalsozialismus. Berlin, New York 2011. Unerlässlich sind die wikipedia-Artikel zu Jaeger wie auch zu den zahlreichen weiter hier behandelten Persönlichkeiten. Mehr zum Thema auch in meinem Beitrag: "Werner Jaegers >Paideiaく. Humanismus, Epistemologie und Zeitgeschichte«, für das 1. Werner-Jaeger-Symposion zur Philosophie und Literatur der Antike vom 26.-28. September 2014 in Nettetal (Niederrhein) die Akten werden auf der Website der Stadt Nettetal publiziert. Einige hier gebotene Überlegungen, Formulierungen und Zitate überschneiden sich mit diesem Papier.
} 
zungen mit dem Humanismus bis heute. ${ }^{2}$ Wir sind uns bewusst, dass, wenn wir heute von Humanismus reden, die Bedeutungen und Gehalte dieses Begriffes noch vielfältiger als damals geworden sind. Ich lege deshalb eingangs Rechenschaft darüber ab, welche grundsätzlichen Punkte ich bei der Lektüre von meines Erachtens wichtigen Publikationen zur Humanismusdebatte beachtet habe: 1) Der Begriff des Humanismus beansprucht hohe Autorität. Er steht für das Ganzheitliche und das Umfassende schlechthin, von dem aus alles Einzelne durch sein Verstehen auf das Gesamte hin seinen Sinn erhält. 2) Zum Begriff des Humanismus gehört die Vorstellung, es gehe um etwas Grundlegendes, das im westlichen Kulturkreis seinen Ausgang genommen habe und in einer Reihe von Erneuerungsbewegungen - Renaissancen, Humanismen - wieder aufgegriffen worden sei und 3) von seinen Vertretern in der Herausarbeitung zeitübergreifender Formen als bestimmend für Mensch und Menschen in der Welt überhaupt erwiesen werden könne. Vom Menschen (homo) und vom Menschlichen (humanum) her würden leitende Kategorien, Prinzipien und Werte entworfen, die eine allgemeine, ja universale Bedeutung beanspruchen könnten. 4) So sei es möglich, in der Kultur generell vernünftige Selbstbestimmung, Autonomie, Rationalität und angemessenes Zusammenleben $\mathrm{zu}$ begründen und $\mathrm{zu}$ vollziehen. 5) Je nachdem beziehen Humanismuskonzeptionen Bereiche ein, die über das Menschliche hinausgehen und doch mit diesem verknüpft sind: Natur, Technik und Göttliches. 6) Die Heraushebung bestimmter Dimensionen des Menschlichen als »klassisch« oder als »leitend « gelten als charakteristisch für die wichtigen geschichtlichen Ausprägungen von Humanismus. Kulturelle Leistungen sowie Erziehung und Bildung sind so zum Inbegriff und Maßstab von Humanismus geworden.

In der Zeit nach 1945 ist der Humanismus mehr und mehr kritisiert worden. Einige setzten zwar nach dem Ende des Zweiten Weltkrieges ihre Hoffnungen gerade in ihn, insbesondere auch in einen christlichen Humanismus. Andere formulierten den Verdacht, er sei Ausdruck einer Fehlentwicklung, ja an den Katastrophen des 20. Jahrhunderts mitschuldig. Ein philosophischer Antihumanismus wurde geradezu zum Programm intellektueller Strömungen. Der Begriff der Bildung, der für den Humanismus so zentral ist, wurde zunehmend in Frage gestellt. Georg Bollenbeck analysierte beispielsweise eindrucksvoll

2 Siehe etwa: Streit um den Humanismus, hg. v. Richard Faber. Würzburg 2003; Julian NidaRümelin: Humanismus als Leitkultur. Ein Perspektivenwechsel, hg. v. Elif Özmen. München 2006; Stefan Kipf: »Moderner Humanismus - Perspektiven und Reflexionen. Festvortrag gehalten am 6.10.2011 anlässlich des zehnjährigen Bestehens des Sächsischen Landesgymnasiums in St. Afra in Meißen«, in: Forum Classicum 4 (2012), S. 248-255. 
Glanz und Elend des deutschen Deutungsmusters »Bildung «. ${ }^{3}$ Schließlich wissen wir alle, dass Post- und Transhumanismus mit Verve als zeitgemäße Trends in Wissenschaft und Kultur verkündet worden sind und noch immer werden. ${ }^{4}$

Umgekehrt fehlt es gleichzeitig nach wie vor nicht an der Aufnahme und Bestärkung des Begriffes im Dienste kultur- und gesellschaftspolitischer Programme. ${ }^{5}$ Erhalten hat sich auch die Vorstellung, man könne die Beschäftigung und Erforschung von Kultur im Rahmen von sogenannten »Humanities« betreiben. Schon Jaeger hat dies betont. In den USA, wo er im Exil lebte, war das bereits damals eine geläufige Bezeichnung der Geisteswissenschaften.

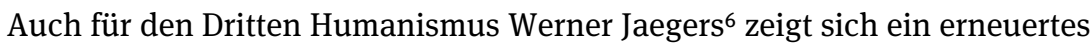
Interesse. Meines Erachtens kann man dazu sicher sagen: Werner Jaegers Werk und seiner Konzeption des Humanismus als neuem »Dritten Humanismus«, in dem die »Paideia«, die griechische Erziehung, so wichtig ist, kommt bei der Verständigung in den Debatten über den Humanismus zweifellos noch immer eine wichtige Funktion $\mathrm{zu}^{7}$ Darüber hinaus hat sein Werk auch, ich sage das nebenbei, im Hinblick auf epistemologische und wissenschaftstheoretische Fragen Bedeutung. Werner Jaegers »Paideia-«konzeption bietet nämlich nicht nur eine Antwort auf die Frage, was Humanismus sei, nämlich eine große geistige Bildungsbewegung, die sich der griechischen Antike verdanke, sondern sie zeigt auch, wie zu wissenschaftlichen Erkenntnissen durch eine philologischhistorische Analyse der antiken Hinterlassenschaft, ihrer Wirkung und Rezeption $\mathrm{zu}$ gelangen ist und welche Folgen die daraus gewonnenen Einsichten für Wissenschaft, Kultur und Gesellschaft auszuüben vermögen. Ihr Humanismus ist zugleich Epistemologie der Humanities: Jaeger legt dar, wie wir erkennen

3 Siehe etwa Günter Seubold: Die Freiheit vom Menschen. Die philosophische Humanismusdebatte der Nachkriegszeit. Darstellung. Analyse. Dokumentation. Bonn 2001; Georg Bollenbeck: Bildung und Kultur. Glanz und Elend eines deutschen Deutungsmusters. Frankfurt a. M., Leipzig 1994; siehe etwa auch Gregor Streim: Das Ende des Anthropozentrismus. Anthropologie und Geschichtskritik in der deutschen Literatur zwischen 1930 und 1950. Berlin, New York 2008, und Reinhardt Koselleck: Begriffsgeschichten. Studien zur Semantik und Pragmatik der politischen und sozialen Sprache. Frankfurt a. M. 2010 (1. Aufl. 2006).

4 Siehe etwa Rosi Braidotti: Posthumanismus. Leben jenseits des Menschen, aus dem Englischen von Thomas Laugstien. Frankfurt a. M. 2014.

5 So Nida-Rümelin: Humanismus als Leitkultur (wie Anm. 2); Humanismus. Sein kritisches Potential für Gegenwart und Zukunft, hg. v. Adrian Holderegger, Siegfried Weichlein und Simone Zurbuchen. Basel 2011.

6 Siehe oben Anm. 1.

7 So etwa Schmidt: »Werner Jaegers >Dritter Humanismus`« (wie Anm. 1), und Jaś Elsner: "Paideia: Ancient Concept and Modern Reception«, in: International Journal of the Classical Tradition 20 (2013), S. 136-152. 
und verstehen können und wie sich daraus eine Theorie von Bildung, Wissenschaft und Kultur ergibt.

\section{Werner Jaeger und die Zeitgeschichte}

Das Verhältnis von Intellektuellen und Zeitgeschichte wie auch der von Wissenschaftlern vertretenen epistemologischen Positionen sind mitbestimmt durch Ideologien und Habitus. Ich führe aus, was ich mit diesen beiden Begriffen bezeichne:

Mit Ideologie meine ich die Ausrichtung auf Vorstellungen, die Intellektuelle entwickeln und verbreiten und denen sich in der Folge eine große Zahl von Menschen verpflichtet fühlen. Die Ideologie löscht die Person des Einzelnen nicht aus, und dies ist an Intellektuellen besonders gut $\mathrm{zu}$ beobachten. Was Einzelne in geduldiger Beschäftigung mit dem Wort über eine längere Zeit hin ausarbeiten, so Werner Jaeger, ist bestens dokumentiert. Ich lese die von ihm verfassten Texte und frage nach der Art und Weise, wie Jaeger als Intellektueller im Spannungsfeld von wissenschaftlichen und politischen Ideologien den $\mathrm{Zu}$ sammenhang dieser Texte und seiner Gegenwart darstellt.

Ich achte dabei auch auf seinen Habitus als Intellektueller (ich übernehme den Begriff von Pierre Bourdieu) sowie seine Stellung und sein Ansehen. Jaeger war einer der führenden deutschen Professoren und der führende Hellenist seiner Zeit. Er produzierte relativ zurückgezogen in seinem Studienzimmer, und wir wissen wenig darüber, was dort geschah, ${ }^{8}$ zugleich war er äußerst aktiv im akademischen Leben, übte großen Einfluss in Gremien, bei Berufungen und über Gutachten und Briefe aus. Er gründete Zeitschriften (so die wichtigste Rezensionszeitschrift im Bereiche der Wissenschaften vom Altertum, den Gnomon), war in Verbänden aktiv und setzte sich für eine Stärkung des humanistischen Gymnasiums und die Erforschung des klassischen Altertums ein.

Nach dem Zweiten Weltkrieg erinnerte man sich nicht selten mit Sehnsucht an seine Zeit zurück. Der Archäologe Ludwig Curtius (1874-1954), der lange Jahre mit einem ähnlichen Lebens- und Arbeitsstil in Rom, seiner zweiten Heimat, gelebt hat, schreibt in seinem autobiographischen Rückblick 1950: In dem "geschlagenen und halb vernichteten Deutschland « sei die »Leidenschaft des geistigen Lebens« sofort nach der Katastrophe wieder aufgeflammt. Dies erinne-

8 Einen Eindruck bietet Karl Hans van Ditzhuyzen: »Begegnung in Boston«, in: Werner Jaeger, hg. v. Manfred Meis und Theo Optendrenk. Nettetal 2009, S. 50-58. 
re ihn an das, was in den 20er Jahren entwickelt worden sei, damals als Werner Jaeger führend gewesen war:

Von ihr (der Leidenschaft des geistigen Lebens) waren auch die Humanisten erfüllt, die 1924 auf einer Tagung in Weimar zur Vertiefung und Verbreitung ihrer Ideen >Die Gesellschaft für antike Kultur`gründeten und von 1925 ab zwei neue Zeitschriften herausgaben [...]. ${ }^{9}$

Sein Engagement aus dem Studierzimmer heraus sah Jaeger als eine Tätigkeit, die er ausgeübt hatte und ausübte als Bürger eines Staates, Deutschlands, aber auch als Angehöriger einer Elite und einer internationalen und vorwiegend männlichen Community, welche sich auf Grund der Urteile ihrer Mitglieder selbst reproduzierte und welche bei ihrer Reproduktion die Anforderungen des Staates und des Bildungssystems berücksichtigte. Der Einfluss bedeutender Lehrer war dabei zentral. Der Lehrer Jaegers war Ulrich von WilamowitzMoellendorff (1848-1931), der größte und einflussreichste Klassische Philologe seiner Epoche. Jaeger wurde sein Nachfolger. Er war weniger einflussreich; doch auch er verfügte über einen bedeutenden Kreis von Schülern, Freunden und Mitarbeitern - auch in Italien. Jaegers Wirkung in den USA ist zwar nicht mit derjenigen vor dem Exil zu vergleichen, sie ist indes auch dort beachtlich geblieben.

Jaeger hat sich kontinuierlich zum grundlegenden Thema des Humanismus geäußert, und zwar in seiner spezifischen Ausprägung als Neuhumanismus. Er hat ihn dann Dritten Humanismus genannt. Die Bezeichnung kommt aus dem Georgekreis und ist wohl bereits um 1900 entstanden. ${ }^{10}$ Jaeger gehörte dem Georgekreis aber nicht an. Der George-Kreis empfing umgekehrt Impulse durch Jaeger. In der Einleitung des 1934 in erster Auflage erschienenen ersten Bandes von Jaegers bedeutenden Werk Paideia kommt die Bezeichnung »Dritter Humanismus« vor. In der zweiten Auflage von 1936 fällt sie weg. Offenbar hat Jaeger die Kritik des befreundeten italienischen Philosophen Guido Calogero aufgenommen, und zweifellos wollte er die Distanz zum »Dritten Reich« deutlich machen. ${ }^{11}$

9 Ludwig Curtius: Deutsche und Antike Welt. Stuttgart 1950, S. 457 f.

10 Schmidt: »Werner Jaegers >Dritter Humanismus « (wie Anm. 1); S. 198; Stiewe, Der »Dritte Humanismus« (wie Anm. 1).

11 Werner Jaeger: Paideia. Die Formung des griechischen Menschen. Bd. 1. Berlin 1934, S. 16 (die gleiche Seitenzahl in der zweiten Auflage von 1936). Die Einleitung trägt den Titel: »Die Stellung der Griechen in der Geschichte der menschlichen Erziehung«. Das Vorwort der ersten 
Jaegers Werk Paideia arbeitet anhand der griechischen Literatur den Gedanken heraus, es gebe ein inneres Bildungsgesetz der griechischen Kultur im Prinzip des Erzieherischen. »Der Grieche« sei ein ursprünglicher Anthropoplast und habe auf allen Gebieten geformt und erzogen, in der Dichtung, der Medizin und Naturwissenschaft, der Ethik und vor allem auch auf dem Bereiche des Politischen. Im Christentum sei dies fortgesetzt worden. Jaeger hat früh vorgehabt, diese Geschichte der griechischen Paideia auch für den Bereich des frühen Christentums darzustellen. Er hat seinen Plan dann auch verwirklicht, allerdings erst in den USA. ${ }^{12}$

1935 verließ er Deutschland zusammen mit seiner Familie, seiner zweiten (jüdischen) Frau und den Kindern, 1936 wurde er aus dem preußischen Staatsdienst entlassen: Er beschreibt den Einbruch selbst in seiner Darstellung der Geschichte der klassischen Philologie an der Universität Berlin von 1870-1945 anlässlich der 150-Jahrfeier der Gründung der Universität im Jahre 1960 folgendermaßen:

Die politische Herrschaft des Nationalsozialismus führte zu Konflikten, die nicht ohne schwerwiegende Folgen blieben, auch für die Berliner Philologie. Es wurde bereits erwähnt, daß der Verfasser dieser Abhandlung sich zu dem Entschluss gedrängt sah, den Lehrstuhl von E. Curtius und Wilamowitz aufzugeben und, wie auch einige der fähigsten jüngeren Köpfe und der verehrte Kollege Professor Eduard Norden, ins Ausland zu gehen. ${ }^{13}$

Zum Hauptanliegen der Berliner Zeit schreibt er 1960:

Die wissenschaftliche Arbeit dieser Jahre hing in der Gesamtheit zusammen mit der Zielsetzung dieser Generation, der Frage nach der Möglichkeit eines neuen Humanismus, der der Schule, Universität und allgemeinen Bildung aus ihrem Ursprung ihren echten Sinn zurückzugeben imstande wäre. Die Einbeziehung des Humanismus in das Geschichtsbild der historisch gewordenen Altertumswissenschaft war der letzte Schritt im Prozesse der

Auflage von 1934 ist mit dem Oktober 1933, dasjenige der zweiten Auflage von 1936 mit dem Juli 1935 datiert. Zu Calogero unten im Abschnitt 4.

12 Siehe etwa Werner Jaeger: Das frühe Christentum und die griechische Bildung, übersetzt v. Walther Eltester. Berlin 1963 (engl. Early Christianity and Greek Paideia, 1961).

13 Werner Jaeger: »Die klassische Philologie an der Universität Berlin von 1870 bis 1945 «, in: Studium Berolinense. Aufsätze und Beiträge zu Problemen der Wissenschaft und zur Geschichte der Friedrich-Wilhelms-Universität zu Berlin, hg. v. Hans Leussink, Eduard Neumann und Georg Kotowski. Berlin 1960, S. 459-485, hier S. 484f. 
Umwandlung der einstigen humanistischen Altertumsstudien. Der Nachweis seiner Verwurzelung in der Struktur des griechischen Geistes selbst wurde in der Paideia erbracht. ${ }^{14}$

Der bedeutende Pädagoge, Philosoph und Psychologe Eduard Spranger (18821963) erklärt das Zustandekommen dieser Zielsetzung im gleichen Band aus der Auseinandersetzung mit dem Historismus. Unter Historismus verstand er die Auffassung, dass im historischen Bewusstsein die geistige Welt erst ihre Gestalt für uns bekomme. Dadurch wurde die geistige Welt zu etwas Relativem. Dieses historische Bewusstsein und die durch dieses im Bestreben nach Verlebendigung heraufgebrachten lebensfeindlichen Relativierungen seien in der ersten Hälfte des 20. Jahrhunderts zum »weltanschaulichen Hauptproblem« geworden. ${ }^{15}$

Jaeger begann mit dem Kampf gegen den Historismus, gegen die Krise und für die Erhaltung der humanistischen Bildung bereits vor seiner Berliner Zeit. Und er setzte in der Hauptstadt Deutschlands und danach im Exil diesen Kampf fort, einen Kampf gegen eine Krise der Kultur, eine Krise der Wissenschaft, insbesondere der Geisteswissenschaft und der humanistischen Tradition.

Das Pathos des Kämpfens gegen behauptete Krisen wird man im historischen Rückblick als Stilisierung und Inszenierung sehen, wie sie zum Habitus eines im höchsten Rang der bürgerlichen Bildungseliten angelangten Aufsteigers passen. Die Erfahrung sozialer Mobilität sensibilisiert für die Fragilität der eigenen Position. Man möchte Sicherheit, Stabilität und Anerkennung. So spüren Aufsteiger besonderes stark Gefährdungen des gesellschaftlichen Systems durch Veränderungen. Die zumeist konservativen Altertumswissenschaftler, die sich durch ihr Studium und ihre Karrieren an der Universität mühsam einen Platz an der Spitze der Gesellschaft erarbeitet hatten, beobachteten mit Misstrauen gesellschaftliche Neuerungen. Sie fürchteten insbesondere, dass das Studium der Antike an Bedeutung verlieren könnte und Latein und Griechisch weniger gut gelernt würden. So waren sie anfällig für die Gefühle von Niedergang, Untergang und Krise. In diesen Kreisen zirkulierte unter anderem auch die Geschichtsphilosophie Oswald Spenglers: Offenbar, so empfanden es viele, war der »Untergang des Abendlandes« nicht mehr fern.

Schaut man auf das, was Kultur und Wissenschaft generell im 20. Jahrhundert gewonnen haben, wird man nicht von einer reinen Krise sprechen können. Zwar zerstörten Faschismus und Nationalsozialismus wichtige Bereiche von

14 Ebd., S. 482.

15 Eduard Spranger: »Das Historismusproblem an der Universität Berlin seit 1900«, in: Studium Berolinense (wie Anm. 13), S.425-443, hier S. 426. 
Kultur und Wissenschaft. Sie blockierten auch viele Entwicklungen. Doch Faschismus und Nationalsozialismus waren auf Kultur und Wissenschaft angewiesen. Sie haben sie gefördert, ihnen eine Aufwertung in ihrem Sinne verliehen und sie für ihre Zwecke eingesetzt. Ideologen, welche dem schlimmsten Irrationalismus huldigten, stützten sich auf die Autorität einer ihnen dienstbaren Wissenschaft.

Im Verständnis Jaegers diente sein Kampf einer Erneuerung. »Krise« und »Dekadenz« sollten so überwunden werden. Im Rückblick formuliert Jaeger die damals von ihm und und »seiner Generation« gestellte Aufgabe so:

Aus solcher Besinnung erwuchs meiner Generation die Aufgabe, nicht nur praktisch für die Existenz des sogenannten Humanismus zu kämpfen, sondern auch ihr Forschen dem Problem zuzuwenden, wie denn die seit zwei Jahrtausenden immer wieder als Tatsache sich bestätigende erzieherische Macht der Antike, auf der die Einheit der abendländischen Welt beruht, aus dem innersten Wesen und der Eigenart der griechischen Geistesform selbst zu begreifen ist. ${ }^{16}$

Jaeger vermochte $\mathrm{zu}$ begeistern und junge Anhänger $\mathrm{zu}$ gewinnen. $\mathrm{Zu}$ ihnen zählte beispielsweise Wolfgang Schadewaldt (1900-1974). ${ }^{17}$

Bereits vor dem Ersten Weltkrieg hatte Jaeger seinen Weg bereitet. In der Basler Antrittsvorlesung von 1914 wird der neue Ton deutlich: Philologie sei »das historisch-genetische Erkennen der antiken Kultur durch Interpretation der Überlieferung und Rekonstruktion der fehlenden Glieder in der Kette des Werdens ${ }^{18}$ Es geht ihm um einen philosophisch-systematisierenden Ansatz und die Suche «nach dem Kraft- und Wertzentrum der philologischen Tätigkeit«. ${ }^{19}$ Er nimmt Nietzsche auf, will über Wilamowitz hinaus und gegen den Historismus und Positivismus antreten.

In Vorträgen hat er dies immer wieder ausgeführt und diese 1937 gesammelt als »ideelle Einheit« unter dem Titel Humanistische Reden und Vorträge noch einmal drucken lassen (2. erweiterte Auflage 1960). Es war, wie er es in einem Brief an seinen Lehrer Hermann Diels (1848-1922) vom 9. Mai 1918 genannt hat, das Zeugnis des »quälenden Ringens der neuen Generation um den Sinn und die Richtung aller Arbeit«.

16 Werner Jaeger: Humanistische Reden und Vorträge. Leipzig, Berlin 1937 (2. erweiterte Auflage 1960), S. XXVI.

17 Siehe Schadewaldt: Gedenkrede auf Werner Jaeger (wie Anm. 1), S. 6.

18 Jaeger: Humanistische Reden und Vorträge (wie Anm. 16), S. 9.

19 Ebd., S. 14. 
Jaeger stand nicht alleine. Zahlreiche bedeutende Persönlichkeiten und Wissenschaftler suchten nach Antworten auf die von ihnen immer wieder beschworenen Krisen. Eduard Spranger entwickelte eine Philosophie der Bildung. Im George-Kreis ließ man sich von der großen Dichtung und von George inspirieren. Man suchte nach geisteswissenschaftlicher Vertiefung. Heidegger ging es um die Aufdeckung des Seins.

Aber auch viele junge Wissenschaften boten Antworten, so mit neu entwickelten generalisierenden Konzepten für die Erfassung von allgemein Wichtigem. Und auch sie wollten eine Antwort auf die Probleme der Zeit geben und die gesellschaftliche und politische Relevanz von Wissenschaft verbessern. Soziologie, Ethnologie und Wirtschaftswissenschaften sind zu nennen. Werner Jaeger freilich schenkte ihnen wenig bis gar keine Aufmerksamkeit - er konzentrierte sich auf seinen eigenen Ansatz.

\section{Jaegers Humanismus und die Ansprüche seiner Humanismuskonzeption}

Jaegers Überlegungen entwickelten sich unter anderem im Zusammenhang der Diskussionen über die Reform des Gymnasiums. ${ }^{20}$ Wie bereits im 19. Jahrhundert waren Bildungs- und Schulfragen stark politisiert. Die konservative Rechte trat eher für den Erhalt des Gymnasiums ein und warnte aber vor »kosmopolitischen« Inhalten der altertumskundlichen Fächer. Die Sozialdemokratie befürwortete eine Einheitsschule. Wer zum linken Parteispektrum zählte, hielt tendenzmäßig die Abschaffung des altertumskundlichen Unterrichts für angezeigt. 1924/25 brachten die Reformen des preußischen Ministerialbeamten Hans Richert (1869-1940) die »Deutsche Oberschule« neben den bisherigen 1900 geschaffenen Typen des Humanistischen Gymnasiums, des Realgymnasiums und der Oberrealschule. Intensiv debattierte man auch über die Frage, welche Texte aus dem antiken Schrifttum ausgewählt werden müssten, was »in sich wertvoll« sei und wo »überzeitliche Werte« verkörpert waren. Jaeger bot hier Antworten. Da ähnliche Debatten auch in Italien stattgefunden haben - ich komme im nächsten Abschnitt darauf zu sprechen -, bekam Jaeger auch dort viel Aufmerksamkeit.

20 Siehe dazu etwa Hans Jürgen Apel und Stefan Bittner: Humanistische Schulbildung 18901945. Anspruch und Wirklichkeit der altertumskundlichen Unterrichtsfächer. Köln, Weimar, Wien 1994. 
Jaegers Philologie war Humanismus. Diesen Humanismus verstand er als Zuwendung zur geistigen Bildung. Jaeger achtete bei seiner Beschäftigung mit der antiken Hinterlassenschaft darauf, wie die Griechen erzogen hatten und sich dadurch wieder erziehen ließ. Von daher waren Autoren wie Homer, Platon, Aristoteles und die Tragiker besonders wichtig, gleichfalls die römische und christliche Rezeption des griechischen Erbes.

Sein Dritter Humanismus zeigt indes auch, wie die Wissenschaften vom Altertum gegliedert werden konnten. Er hat Bedeutung für die Konzeption der Wissenschaften vom Altertum und darüber hinaus für die Geisteswissenschaften. Er gliedert sie. Er ist eine Art Wissenschaftstheorie, die der Philologie, und zwar der Klassischen Philologie, eine Vorrangstellung gibt, aber zugleich die geschichtliche Betrachtung und die Philosophie einbezieht. In Anknüpfung an die bedeutenden wissenschaftstheoretischen Vorgänger wie Friedrich August Wolf (1759-1824) und August Böckh (1785-1867) konzipiert Jaeger ein System der Geisteswissenschaften und der Wissenschaft vom Altertum als Einheit. Die Erkenntnis des Erkannten wird verstanden als ein Verstehen im Nachvollzug der griechischen Paideia. Dies schafft die großen Gemeinsamkeiten, welche die Macht der Geisteswissenschaften rechtfertigt.

Was Jaeger unternommen hat, ist von zahlreichen Wissenschaftlern damals angegangen worden, erinnert sei an den Neukantianismus (von dem sich Jaeger abgegrenzt hat), an Wilhelm Dilthey (1833-1911) oder Ernst Bernheim (18501942), den Verfasser eines grundlegenden geschichtstheoretischen Werkes, das allerdings bei Jaeger nie zitiert wird. Zweifellos hat Jaeger von diesen breiten Anstrengungen viel gelernt und manches übernommen. ${ }^{21}$

Wenig auseinandergesetzt hat er sich - wie schon am Ende des vorangegangenen Abschnittes angetönt - mit den Reflexionen in den modernen Sozialund Wirtschaftswissenschaften. Jaegers Wissenschaftstheorie wirkt antiquiert. Auch ihre anthropologische Ausrichtung führt nicht hin $\mathrm{zu}$ den Wegen, die nach dem Zweiten Weltkrieg dann so wichtig geworden sind. Ähnliches gilt für Jaegers Interesse am Politischen: Wenn Jaeger bei der Herausarbeitung der fundamentalen Bedeutung der griechischen Paideia deren politische Bedeutung so stark unterstrichen hat, so hat er doch keine modernen politikwissenschaftlichen Ansätze rezipiert.

Dass die Klassische Philologie die Leitwissenschaft bleiben sollte, ließ sich erst recht nicht halten. Die modernen Sprachen wurden wichtiger. Und in den

21 Follak: Der »Aufblick zur Idee« (wie Anm. 1), denkt vor allem an Einflüsse aus der Germanistik. 
modernen Philologien wurden denn auch rascher die neuen Ansätze der Postmoderne und der Kulturwissenschaft aufgenommen.

\section{Das Aufkommen des Faschismus und die Frage nach der Bedeutung des Studiums des Altertums in der Gegenwart: Ein Blick auf die 20er Jahre}

Als Jaegers Bewegung des Dritten Humanismus blühte, war die deutsche und deutschsprachige Altertumswissenschaft führend. Die Verbindungen und der Austausch zwischen Italien und Deutschland waren intensiv. Jaegers Lehrer Ulrich von Wilamowitz-Moellendorff war in Deutschland wie in Italien die größte Autorität im Bereiche der Klassischen Philologie und ist in Italien auch persönlich aufgetreten. Der bedeutende italienische Klassische Philologie Giorgio Pasquali (1885-1952) lernte die Universitätslandschaft im Norden bereits früh gut kennen; er hatte in Basel und vor allem Göttingen studiert. Er spielte unter anderem für das für Jaeger so zentrale Gregor von Nyssa-Editionsprojekt eine gewisse Rolle. Einer der wirkungsreichsten Altertumswissenschaftler in Italien war der deutsche Althistoriker Karl Julius Beloch (1854-1929). Seine Schule hat Einfluss auf mehrere Generationen ausgeübt.

Ähnlich wie in Deutschland wurde auch in Italien eine heftige Auseinandersetzung um die Stellung der alten Sprachen und des klassischen Gymnasiums geführt. In beiden Ländern zeichnete sich ab, dass Latein und Griechisch an Bedeutung verloren. Das Fragen nach der Art und Weise des Umganges mit der antiken Kultur in Italien, einem Land mit einer so bedeutenden humanistischen Tradition, war - wie in Deutschland - mit nationalen und nationalistischen Anliegen verzahnt. Die Erinnerung an die Krise von 1914, die Konflikte zwischen Neutralisten und Interventionisten sowie dann vor allem das Aufkommen des Faschismus: Alle diese politischen Themen sind bei der Lektüre der damaligen Publikationen über Bildung und Wissenschaft spürbar. Hinzu kam wie in Deutschland die Auseinandersetzung mit dem Positivismus und mit der Frage nach der Stellung der Geisteswissenschaften. Nicht selten haben italienische Wissenschaftler eine Tendenz der deutschen Altertumswissenschaft zum Positivismus beklagt. Sie riefen nach einer Wissenschaft und Bildung, die lebensnah und nützlich für die historische und politische Erziehung in Italien sein sollten. Solche Anliegen sind durch den Historiker Ettore Pais (1856-1939) 
oder den Klassischen Philologen Ettore Romagnoli (1871-1938) formuliert worden. Nationalismus, wenn nicht gar Chauvinismus, waren fest verankert. Romagnoli hat so Girolamo Vitelli (1849-1935) und dessen Schüler Pasquali der Germanophilie beschuldigt. Gerade der aufkommende Faschismus schien zu versprechen, Italien im Sinne einer Anknüpfung an die antiken und humanistischen Traditionen zu stärken und zu erneuern. Die Schul- und Hochschulreform des jungen neuidealistischen Philosophen Giovanni Gentile (1875-1944), der 1922 Erziehungsminister Mussolinis wurde, schien solche Erwartungen vollständig zu erfüllen. Allerdings musste er bereits 1924 zurücktreten. Auch Werner Jaeger beurteilte die Gentile-Reform in einer Pasquali-Rezension im Gnomon 1934 positiv. ${ }^{22}$

Gentile blieb auch nach seinen Rücktritt aktiv und einflussreich, gehörte von 1925 bis 1929 dem Faschistischen Großrat an und leitete von 1925 bis 1937 das Istituto Nazionale di Fascista di Cultura. 1943 wurde Gentile Präsident der Reale Accademia d'Italia. Weitere Intellektuelle, welche die Antike dem Faschismus dienstbar machen wollten, sind zahlreich. Eine herausragende Rolle kam Giuseppe Bottai (1895-1959) zu, der das Potential moderner Kultur wie auch der Antike und des Humanismus energisch und im Sinne des Faschismus nutzen wollte. 1936 bis 1943 war er Erziehungsminister. Im Herbst 1942 ließ er in Berlin das Institut Studia Humanitatis gründen.

Für die Ideen Jaegers gab es in Italien einen guten Nährboden. Benedetto Croce (1866-1952) hat ihn mit Interesse gelesen. Er rezensierte Jaegers Die geistige Gegenwart der Antike (Berlin 1928) und zeigte sich geradezu begeistert. Jaeger sei es gelungen, eine ewige Lebendigkeit der Antike zu zeichnen. Er erklärte sich damit einverstanden, dass Jaeger der Krise der Gegenwart eine Kraft entgegensetzen wollte. Wenn Jaeger indes die Unfähigkeit des Bürgertums als verantwortlich für die Krise der Gegenwart ansehe, so sei daran zu erinnern, dass die Geistigkeit nicht durch eine ökonomische oder soziale Klasse vertreten werde, sondern durch die Überklasse der gebildeten Eliten. ${ }^{23}$

Croce hatte 1925 das Manifesto degli intellettuali antifascisti redigiert. Es war eine Antwort auf das Manifest von Giovanni Gentile. Giorgio Pasquali hatte das Manifest Croces unterzeichnet. $\mathrm{Zu}$ den Unterzeichnern gehörte auch der Philosoph Rodolfo Mondolfo (1877-1976). Jaeger und Mondolfo standen im Aus-

22 Werner Jaeger: Scripta minora I-II. Roma 1960, II, S. 134.

23 Benedetto Croce: »Gli antichi e noi [1930]«, in: Conversazioni critiche, serie quarta. Bari 1932, S. 283ff.; vgl. Domenico Conte: Weltgeschichte und Pathologie des Geistes. Benedetto Croce zwischen Historischem Denken und Krise der Moderne, aus dem Italienischen übersetzt v. Charlotte Voermanek. Leipzig 2007, S. 100f. 
tausch miteinander. ${ }^{24}$ Mondolfo musste Italien wegen den Gesetzen gegen die Juden 1939 verlassen und lebte fortan in Argentinien.

\section{Jaegers Paideia in den Jahren um 1933}

Am 14. September 1930 wurde die Nationalsozialistische Partei bei den Wahlen in Deutschland mit über sechs Millionen Stimmen und 107 Abgeordneten die zweitstärkste Partei im Reichstag. Jaeger führte in diesem Jahr die Naumburger Tagung über das Klassische durch. Bei den Wahlen vom 31. Juli 1932 erhielt die NSDAP bereits die relative Mehrheit. Am 30. Januar 1933 wurde Adolf Hitler deutscher Kanzler. Die Ideologie des Faschismus zeitigte in einem weiteren Land unheilvolle Erfolge, doch nun mit weit verhängnisvolleren Folgen. Denn Deutschland war grösser und mächtiger als Litauen, Ungarn und Portugal, wo unter Antanas Smetona, Miklós Horthy und Antonio de Oliveira Salazar diktatorische politische Systeme nach dem Vorbild des italienischen Faschismus eingerichtet worden waren.

Sich auf die Antike zu beziehen, um ein autoritär-antidemokratisches Regime zu stützten, war damals nicht ungewöhnlich und konnte an Vorbilder bereits im 19. Jahrhundert anknüpfen. Sowohl im Bonapartismus wie auch im Wilhelminismus war das vorgekommen. In der Epoche des Faschismus ist das Phänomen in Deutschland und Italien am ausgeprägtesten. Doch ist es nicht auf diese beiden Länder beschränkt. So wollte der »Neue Staat« unter Ioannis Metaxas (1936-1941) eine »Dritte griechische Kultur« begründen.

In Deutschland war leicht erkennbar, wie wichtig für Hitler Geschichte und Altertum waren und mit welchem Geschick die NS-Bewegung diese in ihrem Sinne beanspruchen wollten. Viele Althistoriker begrüßten die »Machtergreifung « von 1933. Unter ihnen ragt Wilhelm Weber (1882-1948) heraus. Er sollte in Kürze der einflussreichste Althistoriker werden. Niemand hatte so viele Schüler wie er. Die Ankunft Hitlers in Berlin begeisterte ihn. Er und weitere seiner Kollegen traten mit Publikationen in Erscheinung, in denen sie eine neue Profilierung des Faches Alte Geschichte wie überhaupt der Wissenschaft proklamier-

24 Vgl. Jaeger: Scripta minora (wie Anm. 22), II, S. 407, 411, 413. Im Nachlass Jaegers (in der Houghton Library der Harvard University Cambridge, Mass.) befinden sich mehrere Briefe. 
ten. Vor allem junge ambitionierte Professoren verfassten solche Texte, in denen zu Recht »Programme « NS-affiner Alter Geschichte gesehen worden sind. ${ }^{25}$

Jaeger war zwar fest etabliert, doch er wollte das Humanistische Gymnasium retten. So wurde er einmal mehr aktiv. Er publizierte einen Beitrag in der Zeitschrift Volk im Werden. Sein Dritter Humanismus sollte eine »Brücke« zur Gegenwart sein. Stolz schaute er zurück auf das, was er bisher erfolgreich getan hatte und was in der Weimarer Republik Anerkennung erhalten hatte. So weist er in diesem Text darauf hin, wie er dem humanistischen Anliegen 1924 in seiner Rede zur Reichsgründungsfeier der Berliner Universität Ausdruck gegeben habe. Er zitiert auch noch weitere seiner Publikationen. Immer wieder hebt er die politische Bedeutung des Humanismus hervor. Er endet mit den Worten:

\begin{abstract}
Statt der Verflachung und Materialisierung der echten humanistischen Zucht und Kraft, wie die von uns stets bekämpfte Schulreform von 1924 sie mit ihrem falschen Idol der Kulturkunde in das Gymnasium getragen hat, gebe man der unerlässlichen gründlichen Erlernung der beiden alten Sprachen durch ihre Abzweckung auf eine in diesem echt antiken Sinne sallgemeine Bildung، ihre im nationalen Erziehungsaufbau wieder festverankerte Begründung. ${ }^{26}$
\end{abstract}

Weil es ihm so wichtig war, dass im Gymnasium der Unterricht in den klassischen Sprachen einen festen Platz behalten sollte, beteiligte er sich auch an der Formulierung von Leitsätzen zur Neugestaltung des humanistischen Bildungsgedankens, die der Deutsche Altphilologen-Verband im selben Jahr vorlegte und wo gleichfalls versucht wurde, die klassische Bildung als wichtig für den NSStaat zu erweisen. Der erste Leitsatz lautete:

Das Ziel aller deutschen Erziehung ist der deutsche Mensch als Glied der Volksgemeinschaft. ${ }^{27}$

Das Unternehmen zeitigte höchst zwiespältige Wirkung. Es entstand ein eigentlicher Wettbewerb, wer nun näher beim NS-Staat sei. Ernst Krieck (1882-1947), der Jaeger seine Zeitschrift Volk im Werden geöffnet hatte, distanzierte sich sogleich von diesem. Er, der zuerst wegen nationalsozialistischer Agitation von der akademischen Karriere ausgeschlossen worden war, konnte sich nun etab-

25 Volker Losemann: »Programme deutscher Althistoriker in der >Machtergreifungsphase««, in: Quaderni di Storia 11 (1980), S. 35-105.

26 Werner Jaeger: "Die Erziehung des politischen Menschen und die Antike«, in: Volk im Werden 1 (1933), S. 43-49 (auch als Separatdruck), hier S. 49.

27 Der Text findet sich im 2. Heft der Mitteilungen des Deutschen Altphilologen-Verbandes von 1933 wie auch in der Zeitschrift Humanistisches Gymnasium 44 (1933), S. 209-211, hier S. 209. 
lieren, unter anderem auch durch die Zustimmung des Klassischen Philologen und Jaeger-Anhängers Otto Regenbogen (1891-1966). Da war es naheliegend, Jaeger anzugreifen und zu zeigen, wie viel stärker und nationalsozialistischer die eigenen Vorstellungen waren. Viele andere folgten. Besonders krass äußerte sich der Altphilologe Hans Drexler (1895-1984) in einer Streitschrift gegen Jaeger. Drexler wurde 1943 Rektor in Göttingen, ein Jahr zuvor erschien die zweite Auflage seiner Abrechnung mit Jaeger.

Jaeger hatte - wie bereits erwähnt - indes bereits 1935 Deutschland zusammen mit seiner zweiten Frau Ruth Heinitz verlassen. Ruth Heinitz, Schwester des Juristen Ernst Heinitz, des späteren Rektors der Freien Universität Berlin 1961-1963, stammte aus einer jüdischen Familie. Jaeger benutzte seine Kontakte in die USA, wo er 1934 als erster deutscher Altertumswissenschaftler »Sather Professor « in Berkeley war und Vorlesungen über Demosthenes hielt.

Viele Freunde hielten in Deutschland weiterhin zu Jaeger. Zumeist trafen sie dann auch Sanktionen des NS-Staates, von dem sie sich freilich ähnlich wie Jaeger nicht selten zu wenig klar distanzierten. Dies gilt etwa für den eben erwähnten Otto Regenbogen oder den damals in Schulpforta tätigen Klassischen Philologen Friedrich Müller (1900-1975). Erfolgreich durch ihre Anpassung geblieben sind etwa Viktor Pöschl (1910-1997) oder Richard Harder (1896-1957). Der Mitstreiter und Freund Jaegers Julius Stenzel (1908-1935) starb vorschnell an einer Krankheit. Traurig hat Werner Jaeger damals geschrieben:

Wenn jedes aus dem gewohnten Lebenskreis scheidende Individuum eine spürbare Leere hinterläßt, wieviel mehr ist dieses Gefühl im Recht beim Todes eines Forschers von so reicher Originalität [...].28

Der bekannteste mit Jaeger ähnlich Gesinnte und diesem Verbundene in Deutschland war Eduard Spranger. Spranger emigrierte innerlich, hatte sich aber zuvor höchst ambivalent über den Nationalsozialismus geäußert. Zu erinnern ist sodann an den preußischen Minister Johannes Popitz, der ab 1937 zum Widerstand wechselte. Wolfgang Schadewaldt, Bernhard Schweitzer und Johannes Stroux übernahmen 1937 die Leitung der Zeitschrift Antike und schrieben damals an die Leser:

Über alles Einzelne hinaus leitet uns die Zuversicht: wer heute zu Deutschen von der Antike spricht, kann nichts ins Leere reden. Denn griechisches Wesen, im gemeinsamen Ursprung mit unserem eigenen Dasein verwachsen und im Gang unserer Geschichten immer neu ersehnt und ergriffen, ist in seiner Tiefe Leben von unserem eigenen Leben. Möge die

28 Werner Jaeger: »Julius Stenzel †«, in: Gnomon (1936), S. 108-112, hier S. 108. 
Zeitschrift mitschaffen helfen an dem sich immer erneuernden Bilde der antiken Kultur, so wie die verpflichtende Größe der Tradition deutscher Altertumswissenschaft und das lebendige Zukunftsstreben der Nation es fordern. ${ }^{29}$

Von den Gegnern des Nationalsozialismus kritisierte der Hamburger Altphilologe Bruno Snell (1896-1986) Werner Jaeger. Er warf ihm sowohl die Annäherung an den Nationalsozialismus wie auch das Ästhetische, akademisch Elitäre, in Wahrheit Unpolitische vor: Jaegers Humanismus sei bloße Haltung, nur Ethos, nur Bildung, aber weit weg vom Handeln und so »unpolitisch«. Er könne jeder Politik zugutekommen. ${ }^{30}$ Ähnlich hatten der nationalsozialistische Pädagoge Ernst Krieck sowie die dem Nationalsozialismus gleichermaßen aufgeschlossenen Altertumswissenschaftler Helmut Berve (1896-1979), ein Althistoriker, und der Altphilologe Hans Drexler argumentiert. Berve war der Dritte Humanismus zu lebensfremd, zu wenig vital. ${ }^{31}$

In Italien war Jaegers Wirkung ebenfalls zwiespältig. Zunächst einmal war sie positiv und unauffällig. Der junge Jaeger hatte in Italien gearbeitet, und zwar sehr gut und effizient. Damals geschaffene Beziehungen haben ein Leben lang gehalten. Jaeger war dann in den 20er Jahren in Italien kaum präsent. Zweifellos war indes sein Ruf sehr gut. Denn Wilamowitz, der Lehrer und Vorgänger Jaegers in Berlin, und überhaupt die deutsche Altertumswissenschaft genossen - wie bereits erwähnt - in Italien ein großes Renommee.

Was geschah nun mit dem Aufkommen des Faschismus? Ein wichtiger Faktor sind zweifellos das Verhalten und die politischen Sympathien deutscher Altertumswissenschaftler in Deutschland, zu denen Jaeger Kontakte hatte. Unübersehbar setzten einige prominente Persönlichkeiten unter ihnen ihre Hoffnungen auf Mussolini. Ein Freund Jaegers, der Klassische Archäologe Ludwig Curtius (1874-1954), zählt zu ihnen.

Die Beziehung Jaeger - Curtius hängt mit Jaegers Renommee bei den Klassischen Archäologen zusammen. Jaeger hatte zusammen mit dem führenden Klassischen Archäologen Gerhard Rodenwaldt (1886-1945) die Zeitschrift Die Antike gegründet. Die sogenannte Strukturforschung nahm Anliegen Jaegers

29 Antike 13 (1937), S. 78.

30 Bruno Snell: Rezension Jaeger 1934, in: Göttingische Gelehrte Anzeigen 197 (1935), S. 329353 (= Gesammelte Schriften. Göttingen 1966, S. 32-54.

31 Helmut Berve: »Antike und nationalsozialischer Staat «, in: Vergangenheit und Gegenwart 24 (1934), S. 257-272; wiederabgedruckt und zitiert nach: Über das Studium der Alten Geschichte, hg. v. Wilfried Nippel. München 1993, S. 283-299, hier S. 290ff. Vgl. dazu u. a. Losemann: »Programme deutscher Althistoriker in der >Machtergreifungsphase« (wie Anm. 25), oder Stiewe: Der »Dritte Humanismus» (wie Anm. 1), S. $297 \mathrm{f}$. 
auf. Es handelt sich um eine Richtung, welche sich auf die Form- und Gestaltanalyse von Kunstwerken und das Arbeiten in Bibliothek und Studienzimmer konzentriert hat. Ihr zugehörig war unter anderen Bernhard Schweitzer (1892-1966), der stärker und länger als Curtius und Jaeger vergeblich hoffte, die Wissenschaft vom Altertum mit Hilfe des nationalsozialistischen Staates stärken zu können.

Curtius wurde 1928 wissenschaftlicher Direktor der Abteilung Rom des Deutschen Archäologischen Instituts. Im Rückblick schreibt er darüber:

Die Aufforderung, die Leitung des römischen Instituts zu übernehmen, lockte mich weniger wegen der damit gestellten gelehrten, als wegen der humanistischen Aufgabe. ${ }^{32}$

Er hoffte im Engagement für den Humanismus auch auf Gentile:

Ich hatte 1925 den Philosophen Gentile, den eigentlichen Theoretiker des Faschismus, der einige Jahre Kultusminister war, gebeten, mich in seine ,Philosophische Gesellschaft einzuführen [...].33

Letztlich wurde er enttäuscht, doch die Einsicht kam zu spät:

Der Faschismus war eine rein pragmatische, auf die Omnipotenz des Staates und seines Duce ausgerichtete Parteiherrschaft. ${ }^{34}$

Nach dem Krieg beklagte Curtius die Ungeistigkeit des Faschismus. Seinen Opportunismus und seine früheren Hoffnungen wollte er nicht mehr recht zugeben. Am 18. Januar 1934 hatte Curtius eine Audienz bei Mussolini erhalten. Am 15. Dezember des Vorjahres trug er in Köln im Deutsch-Italienischen Kulturinstitut über »Mussolini und das antike Rom« vor. Die Wiederentdeckung des römischen Altertums durch den Faschismus beurteilte er damals als positiv, obschon Mussolini als »Archäologe« wütete und vieles zerstörte. ${ }^{35}$ Curtius

32 Curtius: Deutsche und Antike Welt (wie Anm. 9), S. 459.

33 Ebd., S. 494.

34 Ebd.

35 Zum Vorgang vgl. Wolfgang Schieder: Mythos Mussolini. Deutsche in Audienz beim Duce. München 2013, S. 118f. Siehe zu Curtius auch: Streit um den Humanismus (wie Anm. 2), S. 157221; Angelos Chaniotis und Ulrich Thaler: "Altertumswissenschaften «, in: Die Universität Heidelberg im Nationalsozialismus, hg. v. Wolfgang Uwe Eckart, Volker Sellin und Eike Wolgast. Heidelberg 2006, S. 391-434, hier S. 391; Marie Vigener: Das Deutsche Archäologische Institut zwischen Wissenschaft, Politik und Öffentlichkeit, 1918-1954. Rahden/Westf. 2012, S. 33ff., 38, 46ff., 65f., 74, 81. 
konnte sich bei seiner Audienz bei Mussolini über Gundolfs Caesar sowie über das Etruskische unterhalten. ${ }^{36}$

1937 wurde Curtius entlassen. Er beschreibt den Vorgang folgendermaßen:

An einem Septembermorgen 1937, als ich als Gast meines Freundes Karl Jaspers in Heidelberg eben mich anzog und mich bei dem Blick durchs Fenster auf den ansteigenden Schloßberg von Heidelberger Erinnerungen bestürmen ließ, klopfte es an meine Tür. Ich öffnete. Draußen stand der Briefträger und brachte mir ein versiegeltes Schreiben. Dieses sprach mit der Unterschrift `des Führers` meine Entlassung aus dem Dienste des Reiches aus. Ich empfand es wie ein Ritterschlag.

Nun wieder zurück zu Jaeger: Auch er hoffte ähnlich wie Curtius eine Zeitlang auf Italien, und wie Curtius verstand er sich als loyalen Staatsdiener. Bei ihm ging es etwas schneller bis zum Bruch, und für ihn wurden die USA wichtig. Am 24. März 1933 hielt Jaeger am Istituto Italiano di Studi Germanici einen brillanten Vortrag, der 1935 in den Studi Germanici gedruckt erschien. Der Titel lautet: "L'umanesimo e le moderne scienze dello spirito«. Jaeger beginnt mit einer captatio benevolentiae: Das unerschöpfliche Thema Humanismus, das so wichtig für die Philologie und die Geistesgeschichte Europas sei, habe seinen Ursprung auf den sieben Hügeln Roms. Er legt dann die Konzeption seines Dritten Humanismus eindringlich dar.

Gleichzeitig wurde seine Paideia übersetzt. Die Einleitung erschien 1935 in der Zeitschrift La Nuova Italia in Florenz. Der Verlag La Nuova Italia war von Elda Bossi und Giuseppe Maranini, ihrem Mann, 1926 in Venedig gegründet worden. Seit 1930 findet man den Verlag unter der Leitung von Ernesto Codignola (1885-1965) in Florenz. Codignola zählte zu den Unterzeichnern des Manifesto degli intellettuali fascisti. Der Übersetzer Luigi Emery (1893-1979) hatte sich 1927 geweigert, der faschistischen Partei beizutreten. Er war Journalist und musste wegen seiner Distanz zum Faschismus ausweichen; er arbeitete dann in Berlin. Er hat zahlreiche deutsche Autoren übersetzt.

Ein Schüler des großen Philologen Giorgio Pasquali, Alessandro Setti, ${ }^{37}$ übernahm die weitere Übersetzung. Es braucht hier nochmals ein paar Informationen zu Pasquali und seiner Stellung in der Wissenschaft, um die Bedeutung dieses Vorganges besser beurteilen zu können. Pasquali hatte das CroceManifest unterzeichnet. Pasquali kannte - wie bereits erwähnt - Deutschland

36 Curtius: Deutsche und Antike Welt (wie Anm. 9), S. $497 f$.

37 Enzo Degani: "La filologia greca in Italia nel cecolo XX«, in: La filologia greca e latina nel secolo XX. Atto del Congresso Internazionale. Roma, Consiglio Nazionale delle Richerche, 17-21 settembre 1984. Bd. 2. Pisa 1989, S. 1135. 
sehr gut. Er hatte dort studiert und gearbeitet. Seine Publikationen waren im deutschsprachigen Raum geschätzt. Pasquali wurde so auch von Jaeger besprochen. Er hatte gute Beziehungen zu Wilamowitz wie auch zu Jaeger.

In Göttingen wurde er mit einem Ehrendoktorat ausgezeichnet. Aktiv bei dieser Initiative war der dortige klassische Philologe Max Pohlenz (1872-1962). Der Reichskirchenminister Hanns Kerrl sorgte gegen den Willen von Pohlenz und weiterer Fakultätsmitglieder für die Auszeichnung eines weiteren italienischen Altertumswissenschaftlers mit einem Ehrendoktortitel, nämlich des in Italien führenden Latinisten Gino Funaioli (1878-1958), von dem Pohlenz indes wenig hielt. ${ }^{38}$

Der konservative, aber dem Nationalsozialismus kritisch gegenüberstehende Pohlenz seinerseits hatte über Antikes Führertum publiziert. Das Werk war in Atene e Roma von Ettore Bignone (1879-1953), dem Direktor der Zeitschrift, einem Klassischen Philologen, der 1938 den Premio Mussolini erhalten sollte, besprochen worden. Bignone war der Auffassung, das Buch habe nichts mit der »modischen« Literatur zu tun, die in Deutschland sich so stark mit dem Konzept der »Rasse « befasse. ${ }^{39}$

Jaegers italienische Paideia erhielt in Italien weit mehr Aufmerksamkeit als die Werke von Pohlenz wie auch weiterer führender deutscher Altertumswissenschaftler. Es wurde mehrfach besprochen, aber nicht ohne Vorbehalte. Der Philosoph Guido Calogeros erachtete die Bezeichnung »Dritter Humanismus» als problematisch. Er kritisierte auch, dass Jaeger so oft von »Führer« und von »Rasse « sprach. ${ }^{40}$ Calogero hat seine Kritik an dieser Begrifflichkeit Jaeger auch brieflich mitgeteilt. Unbeschadet dessen hat er indes dann Jaegers Demosthenes ins Italienische übersetzt. Das Werk erschien 1943. Ich komme unten auch gleich ausführlicher auf diese wichtige Publikation Jaegers zu sprechen.

Der Klassische Philologe Augusto Rostagni (1882-1961) verteidigte Werner Jaeger. Die Rivista di Filologia e di Istruzione classica besprach Jaegers Paideia nicht. ${ }^{41}$ Giulio Augusto Levi (1879-1951), ein bedeutender Kenner des Werkes

38 Cornelia Wegeler: „... wir sagen ab der internationalen Gelehrtenrepublik«. Altertumswissenschaft und Nationalsozialismus. Das Göttinger Institut für Altertumskunde 1921-1962. Wien, Köln, Weimar 1996, S. 231f., sowie Luciano Bossina im vorliegenden Band.

39 Ettore Bignone in: Atene e Roma 3 (1935), S. 215f.

40 Guido Calogero: Rezension des ersten Bandes von Jaegers Paideia in: Giornale critico della filosofia italiana, serie II, 15 (1934), S. 358-371. Vgl. Saggi di etica e di teoria del diritto. Bari 1947, S. 74-105, sowie Scritti minori di filosofia antica. Roma, Bibliopolis [Florenz], 1984, S. 522-546.

41 Emilio Gabba: »Il secondo cinquantennio della >Rivista di Filologia e di Istruzione classica««, in: Rivista di Filologia e di Istruzione classica 100 (1972), S. 442-488, hier S. 462. 
von Giacomo Leopardi, Italianist, Dozent und Literaturkritiker, der bis 1938 in Turin lehren konnte und wegen seiner jüdischen Herkunft zurücktreten musste, besprach das Werk in Atene e Roma und äußerte bei allem Wohlwollen auch Kritik. ${ }^{42}$

Der in Mailand geborene Philosoph Ernesto Grassi (1902-1991), der in Mailand promoviert hatte und $1928 \mathrm{zu}$ Heidegger nach Freiburg ging, wo er - nachdem er vorübergehend bis 1933 in Mailand gelehrt hatte - bis 1938 Italienisch und Philosophie unterrichtete, besprach 1935 Jaegers Werk und Bewegung mit Distanz. Unter anderem warf er dem Dritten Humanismus Vagheit vor. Heidegger stand dem Dritten Humanismus trotz Gemeinsamkeiten kritisch gegenüber. ${ }^{43}$ Wie Heidegger entwickelte Grassi Sympathien für den Nationalsozialismus. Zugleich propagierte er Ideen des faschistischen Erziehungsministers Giuseppe Bottai. Für Bottai war Humanismus ein wichtiges Thema. Es war in seinem Sinne, dass Grassi 1942 in Berlin das Institut Studia Humanitatis gründete. ${ }^{44}$

Grassi hatte auch eine Übersetzung einer Schrift von Giuseppe Bottai besorgt: Verteidigung des Humanismus. Die geistigen Grundlagen der neuen Studien in Italien. Mit einer Einführung von Ernesto Grassi. Jaeger wird dort bewusst nicht erwähnt.

Die Beziehungen Deutschland - Italien blieben wichtig, waren aber nicht immer aufrecht $z u$ erhalten. Für eine Tagung des altertumswissenschaftlichen Kriegseinsatzes vom 2.-3. April 1941 in Berlin wurden laut BA R 4901/2752-A29, Bl. 1_26 vierundreißig Ausländer eingeladen, und zwar zehn italienische Wissenschaftler: Giorgio Pasquali, Giacomo Devoto, Conte Ranuccio BianchiBandinelli, Gino Funaioli, Giulio Gianelli, Paolino Minganzzini, Augusto Rostagni, Ettore Bignone, Achille Vogliano, Luigi Castiglioni. Aber niemand konnte dann anreisen, es blieb bei der Absicht, den Austausch zu pflegen. ${ }^{45}$

42 Giulio Augusto Levi, in: Atene e Roma 3 (1935), S. 177-186.

43 Vgl. Fleming: »Heidegger, Jaeger, Plato: The Politics of Humanism« (wie Anm. 1).

44 Vgl. Wilhelm Büttemeyer: Ernesto Grassi - Humanismus zwischen Faschismus und Nationalsozialismus. 2. Aufl. Freiburg i. Br. 2010.

45 Frank-Rutger Hausmann: [Rez.] Beat Näf: Antike und Altertumswissenschaft in der Zeit von Faschismus und Nationalsozialismus. in: H-Soz-u-Kult, H-Net Reviews. February, 2002. URL: http://www.h-net.org/reviews/showrev.php?id=16520 (eingesehen am 31.10.2016). 


\section{Demosthenes - der Redner und die Politik in Antike und Gegenwart (1938/39): Werner Jaeger, Piero Treves und Arnaldo Momigliano}

Ebenso wichtig wie die dreibändige Paideia ist ein weiteres Werk Jaegers, das in diesen Jahren entstand: Demosthenes. Der Staatsmann und sein Werden. Auch an diesem Buch zeigen sich die Einflüsse der Zeitgeschichte.

Der große athenische Redner Demosthenes war seit jeher eine Gestalt, die dazu diente, sich mit bewegenden Gegenwartsfragen auseinanderzusetzen. Bereits im 18. Jahrhundert benutzte man ihn im Dienste patriotischer Bewegungen. Später bediente man sich seiner, um zu zeigen, wie Intellektuelle sich für einen starken und möglichst großen Nationalstaates mit einer lebendigen und starken nationalen Leitkultur einsetzen konnten. Im Deutschland des 19. Jahrhunderts hatte sich auf dem Hintergrund des Weges zum Nationalstaat die Gleichsetzung Makedoniens mit Preußen etabliert. In Italien dachte man bei Makedonien gerne an Savoyen / Piemont: Demosthenes erschien von daher als rückwärtsgewandter Politiker. Der Makedonenkönig Philipp war der Sieger. Er hatte den großen Nationalstaat geschaffen. Die athenischen Gegenspieler des Demosthenes, Aischines und Isokrates, beurteilte man hingegen als weitsichtig und zukunftsorientiert. Solche Einschätzungen finden sich auch in den einflussreichen Geschichtswerken von Eduard Meyer und Julius Beloch. Im Zuge des Ersten Weltkrieges wurde Demosthenes Inbegriff eines egoistischen und demagogischen politisierenden Advokaten: Die Assoziationen mit Verhältnissen in Grossbritannien waren dabei durchaus gewollt. Andere Wissenschaftler sahen in Demosthenes einen tragischen Helden im Kampf für die Freiheit. Diese Auffassung findet sich auch in einem wirkungsvollen Büchlein des französischen Ministerpräsidenten Georges Clémenceau aus dem Jahre 1925.

Werner Jaegers Demosthenes erschien 1939 in Deutschland. Dieser Publikation vorausgegangen war eine englischsprachige Ausgabe im Jahre 1938. 1943 erschien die oben erwähnte italienische Übersetzung von Guido Calogero. Jaeger lebte nun in den USA. Die Entstehung des Buches beschreibt Jaeger so:

Den äußeren Anlaß zur Abfassung des Buches, das hier zum erstenmal in seiner deutschen Originalfassung erscheint, gab mir die 1932 an mich ergangene Einladung der University of California in Berkeley, die Sather Lectures des Jahres 1934 zu halten. Aber wenn meine Gedanken über Demosthenes auch erst im Jahre 1932-1933 ihre vorliegende Form erhalten haben, sind sie doch weit früher entstanden. Im kommenden Frühjahr ist es 
fünfundzwanzig Jahre her, daß ich meine erste Vorlesung als Professor an der Baseler Universität hielt. Sie hatte Demosthenes zum Gegenstand, und es gibt kein Thema, das ich seither so häufig und von den verschiedensten Seiten in Vorlesungen behandelt habe wie dieses. 46

Jaeger sieht in Demosthenes, und nicht in Philipp, jene positive Gestalt, welche den Gedanken griechischer Kultur und einer griechischen nationalen Einigung vertreten habe.

Den partikularistischen Realpolitiker hat in Demosthenes neuerdings vor allem Arnaldo Momigliano, Contributi alla caratteristica di Demostene, in Civiltà Moderna (Jahrgang 1931) S. 711ff. gesehen. Aber wie Piero Treves, Rivista di Filologia Bd. LX (1932) 68ff. bereits gegen diese Auffassung geltend macht, hat Momigliano Demosthenes zu einseitig von seiner frühen Periode aus verstehen wollen und die Entwicklung nicht genügend gewürdigt, welche er seither unter der Einwirkung des mächtigen historischen Schicksals, das >Philipp « hieß, durchgemacht hat. ${ }^{47}$

Der junge italienische Historiker Piero Treves (1911-1992) war wie der aus einer orthodox-jüdischen Familie des Piemont stammende Arnaldo Momigliano (1908-1987) ein Schüler des bedeutenden Historikers Gaetano De Sanctis (sie studierten bei ihm in Turin), der seinerseits Schüler Belochs und 1929 dessen Nachfolger an der Universität Rom wurde. Der Katholik De Sanctis interessierte sich für Politik. Zu Beginn des Ersten Weltkrieges hatte er sich als Neutralist geweigert, einen Protest gegen Giolitti zu unterzeichnen, der den Frieden wünschte. 1929 verweigerte er den Eid auf das faschistische Regime. Während nun der junge Momigliano Sympathien für den Faschismus zeigte, so auch in der Interpretation des Demosthenes, bezog Treves gegen den Faschismus Stellung und verknüpfte mit diesen politischen Präferenzen auch seine wissenschaftliche Behandlung des Demosthenes, in dem er den Kämpfer für Freiheit und Autonomie herausstrich. Seine Ausführungen, welche auf seine Dissertation zurückgehen, fanden den Beifall von Benedetto Croce. Bei Laterza erschien 1933 sein Buch Demostene e la libertà greca (Bari 1933).

Die Gestalt des Demosthenes hat in den folgenden Jahren wiederholt Aufmerksamkeit gefunden. Unter französischen Althistorikern gab es eine Tendenz, in ihm eine Autorität zu sehen, die vor dem Krieg gewarnt habe und für die Prinzipien der Freiheit eingestanden sei, wie sie der Widerstand gegen Hitler vertrat: In der durchaus renommierten und seriösen britischen Zeitschrift Greece \& Rome erschien im Mai 1941 ein Aufsatz mit dem Titel »Philip >Alias`

46 Werner Jaeger: Demosthenes. Der Staatsmann und sein Werden. Berlin 1939, S. VII.

47 Ebd., S. 248 (Fußnote 41 zu S. 170, Kapitel VII). 
Hitler«. Verfasst hatte ihn Adela M. Adam, die in ihrem Beitrag unter anderem die Bombenangriffe der britischen Luftwaffe auf Deutschland rechtfertigte. ${ }^{48}$

Für die Verbindungen zwischen Gegenwart und Antike waren aber andere Themen viel wichtiger. Die Olympischen Spiele 1936 in Berlin sind zu nennen und die großen Feiern, welche im faschistischen Italien zur Zelebrierung des Augustus und seines Zeitalters durchgeführt wurden.

\section{Werner Jaeger und sein Dritter Humanismus nach 1945}

Jaeger bekam nach 1945 immer wieder Anerkennung: So wurde er 1955 Mitglied im Orden pour le Mérite, im gleichen Jahr erhielt er den Antonio-Feltrinelli Preis. Die Scripta minora wurden in zwei Bänden mit einem eigens verfassten Rückblick als Einleitung 1960 in Rom herausgegeben.

Jaeger hoffte nach wie vor auf die Wirkung des Humanismus. 1948 hob er in einem Brief an Eduard Spranger die Vorbildfunktion der USA heraus. ${ }^{49}$ Mehrere Bücher zeigten, wie der Humanismus gerade bei den Kirchenschriftstellern, allen voran Gregor von Nyssa, vertieft worden war.

Mit zahlreichen Publikationen kämpfte er für seine Ideale. Die Erinnerung an ihn ist aber stark geprägt durch das Scheitern der Bewegung des Dritten Humanismus. Diese kritische Sicht ist nicht zuletzt durch Arnaldo Momigliano (1908-1987) und Moses I. Finley (1912-1986) verbreitet worden.

Der brillante italienische Althistoriker Arnaldo Momigliano hielt seine früh entwickelte Kritik an Werner Jaeger aufrecht und warf diesem nun aber auch noch die Annäherung an den Nationalsozialismus vor. Jaeger erscheint als Vertreter einer falschen Ideologie, von der man sich befreien müsse. Momiglianos Urteil ist besonders folgenreich, weil er $\mathrm{zu}$ jenen überragenden Intellektuellen zählte, die weltweit in den wissenschaftlichen Einrichtungen präsent waren und zugleich Wissenschaftsgeschichte betrieben. Eine ähnliche Persönlichkeit war Moses I. Finley, und Finley teilte das Urteil Momiglianos, nahm es auf und

48 Adela M. Adam: »Philip `Aliasく Hitler«, in: Greece \& Rome 10 (1940/41), S. 105-113.

49 Manfred Overesch: »Dokumentation zur deutschen Bildungspolitik nach dem 2. Weltkrieg. Werner Jaegers Brief an Eduard Spranger vom 26. Mai 1948«, in: Gymnasium 89 (1982), S. 109121, hier S. 117. 
gab es weiter, so in seinem ebenfalls einflussreichen Buch The Use and Abuse of History. ${ }^{50}$

In der Paideia sah Momigliano ein gescheitertes Unternehmen, das kurzfristig wichtig gewesen sei und das keine guten Einflüsse ausgeübt habe. 1967, im Appendix seiner bibliographischen Orientierung als Zugabe zur Griechischen Geschichte von Gaetano de Sanctis, skizziert er die Entwicklungen auf dem Gebiete der Griechischen Geschichte. Man befinde sich im Zeitalter der Dekolonisation. In Italien habe man sich auch an die deutsche Verehrung von nichtübersetzbaren Worten wie Paideia angeschlossen und die Ideengeschichte von der politischen und sozialen Geschichte getrennt. Davon müsse man sich befreien. Henri-Irénée Marrou habe das viel besser gemacht. ${ }^{51}$

Doch Jaeger hat in Italien und Deutschland Anhänger und Leser behalten. Davon zeugt die Bompiani-Ausgabe der Paideia durch Giovanni Reale (19312014), der lange Jahre an der Università Cattolica di Milano Philosophie lehrte.

In der Tat ist Werner Jaeger eine bedeutende Gestalt der Geschichte der Geisteswissenschaften in der modernen Welt. Überdies sind seine Überlegungen als Modell der Verständigung über epistemologische Fragen wichtig. Von daher ist es nicht erstaunlich, dass er im wissenschaftlichen und kulturellen Austausch zwischen Italien und Deutschland in den 20er bis 40er Jahren wie auch deren späterer Rezeption eine wichtige Rolle spielt.

50 Moses I. Finley: The Use and Abuse of History. London 1975, S. 75-86, v. a. S. 78f.: »Werner Jaeger was for my generation the central, and ambiguous, figure in the latest chapter of the story. [...] Momigliano has discussed his significance time and again, though never at length [...] most recently in a lecture on >Perspective 1967 of Greek History ‘ [...], where he briefly noted the programmatic aim of Paideia as an aristocratic idea."

51 Arnaldo Momigliano: "Appendice. Prospettiva 1967 della storia greca«, in: Introduzione bibliografica alla storia greca fino a Socrate. Appendice a Gaetano de Sanctis, Storia dei Greci. Firenze 1967, repr. 1975, S. 165-186 (= Quarto contributo, 43-58), hier S. $176 f$. 
Bereitgestellt von | UZH Hauptbibliothek / Zentralbibliothek Zürich 